Hier können Sie CME-Punkte sammeln a) für die Pflichtfortbildung aller Vertragsärzte und b) für freiwillige Fortbildungszertifikate, die viele Landesärztekammern anbieten.

Die Multiple-Choice-Fragen beziehen sich auf den vorangegangenen Fortbildungsbeitrag (Seiten 42-45). Die Antworten ergeben sich z. T. aus dem Text, z. T. beruhen sie auf medizinischem Basiswissen!
CME-Herausgeber- und Review-Board:

Dr. H. J. K. Barwitz, Prof. Dr. A. Berghaus, Prof. Dr. Dr. h.c. Th. Brandt, Prof. Dr. W. G. Daniel, Prof. Dr. W. Eisenmenger, Prof. Dr. K. Friese, Prof. Dr. H.S. Füessl, Prof. Dr. B. Göke, Prof. Dr. R. Gradinger, Prof. Dr. H. Holzgreve, Prof. Dr. A. Imdahl, Prof Dr. K.-W. Jauch, Prof. Dr. K. Krüger, Prof. Dr. H.-J. Möller, Prof. Dr. D. Reinhardt, Prof. Dr. Dr. h.c. Th. Ruzicka, Prof. Dr. Ch. Stief, U. Weigeldt.

\title{
Fragen zum Thema „Palpitationen in der Brust“
}

\section{- 1. Welchen Anteil am Herzminutenvolu- men hat die Vorhofkontraktion?}
A $0-10 \%$
B $10-15 \%$
C $20-30 \%$
D $50-60 \%$
E $80-90 \%$

\section{Wie viele 85-Jährige haben statistisch gesehen Vorhofflimmern?}
A $1 \%$
B $2 \%$
C $5 \%$
D $8 \%$
E $20 \%$

- 3. Welche anamnestische Befundkonstellation passt zur Diagnose Vorhofflimmern?

A Aufstoßen und Völlegefühl

B Zyanose und Beinödeme

C Antriebslosigkeit und Gewichtszunahme

D Nachtschweiß und retrosternales Brennen

E Herzrasen und Schwindel

\section{- 4. Welcher EKG-Befund ist charakteris-} tisch für Vorhofflimmern?

A Ventrikuläre Extrasystolen.

B Unregelmäßige Vorhoferregung anstelle der P-Wellen.

C Verlängerte PQ-Dauer.

D ST-Streckensenkung.

E Pathologische Q-Zacken.

\section{Was versteht man unter dem Begriff "Lone atrial fibrillation"?}

A Einen alleinstehenden Patienten mit Vorhofflimmern.

B Flimmern nur des linken Vorhofs.

C Vorhofflimmern ohne zugrunde liegende oder assoziierte Erkrankung.

D Eine einmalige kurze Flimmerepisode.

E Vorhofflimmern bei regelmäßigem Sinusrhythmus.

- 6. Welche Gefahr ist vor der Kardioversion von Vorhofflimmern zu beachten?

A Vorhandene Vorhofthromben können in den großen Kreislauf ausgeworfen werden.

B Es kann zur Mitralinsuffizienz kommen.

C Die Maßnahme ist nur selten wirksam.

D Die Zeit drängt, da die Kardioversion nach drei Tagen Dauer des Vorhofflimmerns nicht mehr möglich ist.

E Hautverbrennungen der Brust sind eine häufige Nebenwirkung der Kardioversion.

\section{-7. Bei welchem Patienten mit Vorhof- flimmern ist das Apoplexierisiko am höchsten?}

A Alter 60 Jahre, keine strukturelle Herzerkrankung, keine zusätzlichen kardiovaskulären Risikofaktoren

B Alter 65 Jahre, zusätzlich arterielle Hypertonie und Diabetes mellitus

C Alter 80 Jahre, keine strukturelle Herzerkrankung
D Alter 80 Jahre, zusätzlich Mitralinsuffizienz und Diabetes mellitus

E Alter 80 Jahre, zusätzlich KHK und Diabetes mellitus, unter Antikoagulation

- 8. Welcher INR-Bereich wird einem Patienten mit Vorhofflimmern und Mitralstenose empfohlen?
A $0,5-1,0$
B $1,5-2,0$
C $2,5-2,9$
D $3,0-3,5$
E $3,6-4,0$

- 9. Welche der genannten Substanzen ist bei Vorhofflimmern und bestehendem WPW-Syndrom kontraindiziert?

A Amiodaron

B Flecainid

C Digitalis

D Propafenon

E Betablocker

- 10. Was versteht man unter einem Pulsdefizit?

A Das Fehlen eines peripheren Pulses

B Die Differenz zwischen der Herzfrequenz im EKG und der klinisch gemessenen Herzfrequenz

C Den Unterschied zwischen dem am rechten und linken Arm gemessenen Puls

D Die Differenz zwischen dem auskultierten und dem palpierten Puls

E Einen Herzstillstand

\section{ANTWORTFORMULAR}

Mit Einreichen dieses Fragebogens erkläre ich mich damit einverstanden, dass die angegebenen Daten zum Zweck der Teilnahmebestätigung gespeichert und bei erfolgreicher Teilnahme an den Einheitlichen Informationsverteiler (EIV) der Ärztekammern weitergegeben werden.

Wichtiger Hinweis:

Eine Auswertung ist künftig nur noch möglich, wenn Sie Ihre EFN auf dem Antwortformular angeben!

Nähere Hinweise hierzu unter: www.cme-punkt.de/faq.htm

\section{„Palpitationen in der Brust"}

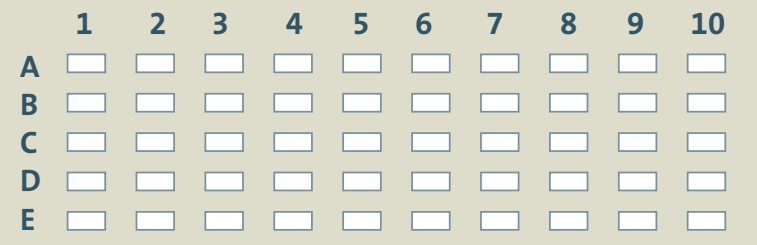

Bitte kreuzen Sie pro Frage je eine Antwort deutlich an.
Name, Vorname Fachrichtung

Geburtsdatum Einheitliche Fortbildungsnummer (EFN)
Und so kommen Sie zu lhren Punkten:

Teilnahme im Internet: unter www.cme-punkt.de. Dor führen wir für Sie ein elektronisches Punktekonto.

Teilnahme per Brief: Fragebogen ausfüllen und mit frankiertem Rückumschlag an Urban \& Vogel GmbH CME MMW-Fortschr. Med. Postfach 81664 München. 